\title{
Vaccine communication in a digital society
}

\author{
Now more than ever before, it is vitally important for scientists, clinicians and policymakers to communicate the \\ significance of vaccination to the public in order to counteract the spread of misinformation.
}

$\mathrm{V}$ accination is demonstrably one of the most effective public health interventions globally, which makes it remarkable and alarming that 'vaccine hesitancy' was named one of the World Health Organization's ten threats to global health for 2019.

A recent report on progress towards measles elimination estimated that 20.3 million lives have been saved by measles vaccination between 2000 and $2015^{1}$. Despite this, immunization uptake is falling and measles caused at least 140,000 deaths worldwide in 2018, with reported measles cases for 2019 the highest in a single year since $2006^{2}$. That children continue to die in the third decade of the twenty-first century from a disease for which we have had an effective vaccine for almost 60 years reflects a crisis in both the delivery of public health interventions and public understanding of science.

High immunization uptake is critical for protecting people from infectious diseases, but barriers to immunization are complex. Affordability and accessibility, complacency around the risks of vaccinepreventable diseases, vaccine confidence and political concerns all contribute to falling immunization rates. While for much of the world's childhood population, access to immunization is the big issue to solve, one key influencer of immunization uptake is false or misleading information, leading to low public confidence in vaccines. In the modern digital world, this is a challenge that requires a global, interdisciplinary approach employing evidence-based communication strategies, but sadly these strategies are often more skilfully exploited by anti-vaccination campaigners than by public health messengers ${ }^{3}$.

It is important for parents to make informed decisions about their children's health, and improved access to health information supports these decisions. However, the current climate of 'new power versus old' often encourages people to challenge experts rather than trust science, and social media platforms provide a tool for anti-vaccination narratives to spread quickly. These 'new power' strategies have created the conditions for vaccine hesitancy to grow, and with no geographical limits, and we've seen immunization rates drop globally ${ }^{4,5}$. Our response as an international scientific community thus far has too often been weak, unintentionally reinforced negative messages and not been credible for a sceptical public.

So, as members of 'old power' institutions (including scientists, medical doctors, public health experts and policymakers), how can we utilize these unfamiliar platforms and tools to overcome this devastating rise of infectious diseases? A key action is to tirelessly provide messages about the evidence and science behind immunization and to create an environment where knowledge of vaccines and their role in health is embedded in our culture, and does not have to be questioned at the end of every needle. Creating a trustworthy information environment that engages evidence-based health communication strategies to provide timely, credible and audience-specific content is critical ${ }^{2}$. We have created the Vaccine Knowledge website (https://www.vaccineknowledge.info), which is the only UK nongovernmental website accredited by the World Health Organization's Vaccine Safety Net, to support this goal, taking a strategic approach to content development. Risk communication approaches utilized are: answering common questions about vaccination and providing harm and benefit information to support 'risk versus benefit' decisions ${ }^{6}$.

An approach often adopted by antivaccination or pro-choice campaigns is the use of personal narratives, and Vaccine Knowledge's personal stories often harbour more interest from media teams than our researchers, reinforcing the switch in public trust from experts to relatable peers.

Scientists and clinicians remain the experts, and we have an obligation to share our knowledge with the public to enable evidence-based decision making. To effectively address misinformation and public concerns, we must adapt to this new era and utilize digital media tools familiar to our fast-moving society. Factchecking initiatives should be supported and encouraged by policymakers and topic experts, scientific articles on vaccine safety should be summarized in an accessible format such as infographics and videos, and shared on social media platforms. A further major concern for scientists is the poor editorial policy of some publishers, which allows publication of manuscripts with overinterpretation of the significance of data to support a spurious concern about vaccine safety. Such publications are picked up by the anti-vaccination community as scientific evidence rather than the bad science that they represent. Despite these exceptions, journals do publish a wealth of science to counteract misinformation, but these data are of little use to address vaccine hesitancy unless they are published in the public sphere. 'Fake news' or false stories are 70\% more likely to be shared than true stories on Twitter ${ }^{7}$, so we must present evidence-based information in an enticing, provocative format to gain equivalent traction.

If we are to overcome this climate of falling vaccine confidence, we must engage alternative partners who are relatable, trusted representatives, and work collaboratively to create an environment in our societies where immunization is accepted as a right for the wider community.

\section{Tonia M. Thomas ${ }^{1,2}$ and Andrew J. Pollard ${ }^{1,2}$ \\ ${ }^{1}$ Oxford Vaccine Group, Department of Paediatrics, University of Oxford, Oxford, UK. ${ }^{2}$ NIHR Oxford Biomedical Research Centre, Oxford, UK.

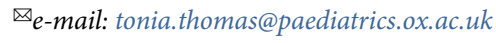

Published online: 24 March 2020 https://doi.org/10.1038/s41563-020-0626-7

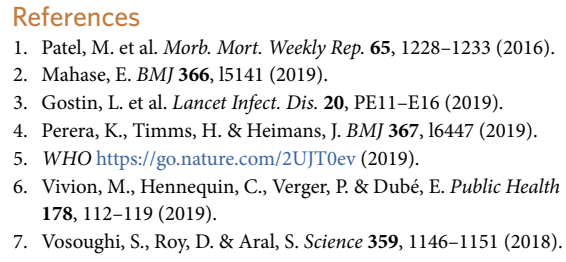
Health Organization's (WHO) Strategic Advisory Group of Experts. A.J.P. is a National Institute for Health Research (NIHR) senior investigator. The views expressed in this article do not necessarily represent the views of the DHSC, JCVI, NIHR or WHO. 